\title{
Asteroseismology of the extreme metal-deficient field high-amplitude SX Phe variable BL Cam
}

\author{
E. Rodríguez, ${ }^{1}$ S. Fauvaud, ${ }^{2,3}$ J. A. Farrell, ${ }^{4}$ A.-Y. Zhou, ${ }^{5}$ J.-P. Sareyan, ${ }^{6}$ \\ M. J. López-González, ${ }^{1}$ G. Klingenberg, ${ }^{7}$ M. Wolf, ${ }^{8}$ A. Rolland, ${ }^{1}$ P. López de Coca, ${ }^{1}$ \\ P. Van Cauteren, ${ }^{9}$ P. Lampens, ${ }^{10}$ M. Helvaci, ${ }^{11}$ E. G. Hintz, ${ }^{12}$ L. Král, ${ }^{13}$ F. Fumagalli, ${ }^{3}$ \\ J. H. Simonetti, ${ }^{14}$ B. H. Granslo, ${ }^{7}$ L. Kotkova, ${ }^{15}$ G. Santacana, ${ }^{2}$ J. Michelet, ${ }^{16}$ \\ H. Kucáková, ${ }^{13}$ R. Kocián, ${ }^{13}$ K. Truparová, ${ }^{13}$ A. Avdibegovic, ${ }^{11}$ M. Blazek, ${ }^{11}$ \\ J. Kliner, ${ }^{11}$ P. Zasche, ${ }^{11}$ M. Vilásek, ${ }^{13}$ S. Bartosíková, ${ }^{13}$ O. Trondal ${ }^{7}$ \\ ${ }^{1}$ Instituto de Astrofísica de Andalucía, CSIC, E-18080 Granada, Spain, E-mail:eloy@iaa.es \\ ${ }^{2}$ Association AstroQueyras, Le bois de Bardon, Taponnat, La Rochefoucauld, France \\ ${ }^{3}$ Groupe Européen d'Observations Stellaires (GEOS), Bailleau I'Evêque, France \\ ${ }^{4}$ Sulphur Flats Observatory, Jemez Springs, NM 87025, USA \\ ${ }^{5}$ National Astronomical Observatories, Chinese Academy of Sciences, Beijing 100012, China \\ ${ }^{6}$ Observatoire de la Côte d'Azur, BP 4229, F-06304 Nice cedex 4, France \\ ${ }^{7}$ Variable Star Section, Norwegian Astronomical Society, N-0315 Oslo, Norway \\ ${ }^{8}$ Astronomical Institute, Charles University Prague, CZ-180 00 Praha 8, Czech Republic \\ ${ }^{9}$ Beersel Hills Observatory, 1650 Beersel, Belgium \\ ${ }^{10}$ Koninklijke Sterrenwacht van België, B-1180 Brussel, Belgium \\ ${ }^{11}$ Ankara University, Department of Astronomy and Space Sciences, Ankara, Turkey \\ 12 Department of Physics and Astronomy, Brigham Young University, Provo, UT 84602, USA \\ ${ }^{13}$ Obs. and Planetarium of Johann Palisa, VSB-Technical University, Ostrava-Poruba, Czech Republic \\ ${ }^{14}$ Martin Obs., Physics Dep., Virginia Polytechnic Institute \& State Univ., Blacksburg, VA 24061, USA \\ ${ }^{15}$ Astronomical Institute, Academy of Sciences, Ondrejov, Czech Republic \\ ${ }^{16}$ Club d'Astronomie Lyon Ampére, 37 rue Paul Cazeneuve, Lyon, France
}

Classical pulsating stars displaying large amplitudes in the Lower Instability Strip commonly are pure monoperiodic or double-mode radial pulsators. Only in a very few cases, some additional independent modes have been detected in their light curves which are preponderantly nonradial. BL Cam is suspected to be one of these very few exceptions. This star is an extreme metal-deficient field high-amplitude SX Phe variable in which multiperiodicity has been claimed by different authors (see Fauvaud et al. 2006 for a review) with the secondary peaks displaying very small amplitudes compared to the main periodicity. Thus, this star promised to be a good target for asteroseismic studies. The main aim of this work is to realize a detailed study of this star concerning: (a) its pulsational content and (b) the behaviour of its main periodicity in the O-C diagram. Preliminary results concerning the former point are presented here.

The observations were carried out between August, 2005 and March, 2006 from a number of observatories in Europe and America, with observations collected during more than 100 nights and 550 hours. All observations were obtained with CCD cameras and various filters, the majority of them using the Johnson $V$ filter. More than 500 new times of light maximum have been also determined. In particular, at Sierra Nevada Observatory (SNO), the data were sequentially collected in the filters BVI on 13 nights and about 70 hours in each filter (Fig. 1). From this, nearly 300 hours of useful data were used for the frequency analysis following the method described in Rodríguez et al. (1998) and Lenz \& Breger (2005).

The results show very dense pulsational microvariability in this star in addition to the high-amplitude main periodicity $\left(\mathrm{f}_{0}=25.5765 \mathrm{~cd}^{-1}, \Delta \mathrm{V}=153 \mathrm{mmag}\right)$. In total, 22 secondary peaks, with very small amplitudes (between 7.4 and $1.6 \mathrm{mmag}$ ), were found significant, corresponding to 21 independent modes and one combination $f_{0}+f_{1}$. This represents the most complex spectrum ever known in a high-amplitude pulsator in the Lower Instability Strip and opens the possibility to investigate similar microvariability features in other classical high-amplitude objects. Moreover, some additional periodicities are probably still remaining 


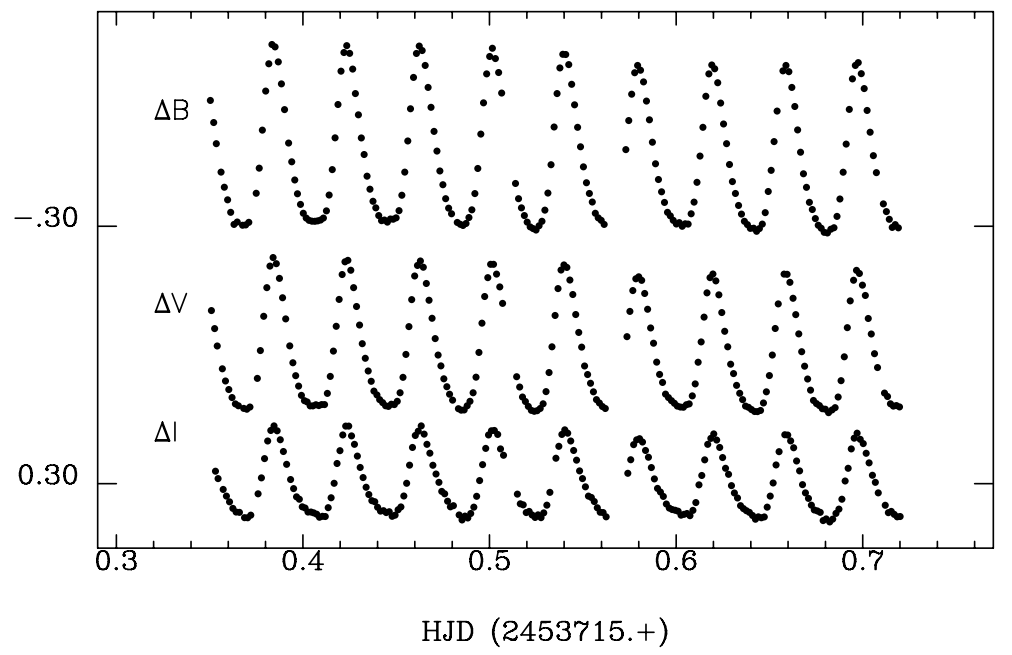

Figure 1: BVI light curves of BL Cam obtained on December 10th, 2005 at SNO.

in the residuals of the frequency spectra. The amplitude of the main periodicity $f_{0}$ seems to be stable during decades but the majority of the secondary modes present strong amplitude changes from one epoch to another.

Multicolour photometry analysis suggests that $f_{0}$ corresponds to the fundamental radial mode whereas $\mathrm{f}_{1}=25.2523 \mathrm{~cd}^{-1}$ is suggested as a nonradial modes with $\ell=1$. Moreover, the large changes occurring in the amplitude of $\mathrm{f}_{6}=32.6464 \mathrm{~cd}^{-1}$ do not support the sometimes claimed idea about this mode being the first overtone of radial pulsation and, thus, BL Cam being a radial double-mode pulsator.

\section{References}

Fauvaud S., Rodríguez E., Zhou A.-Y., et al., 2006, A\&A, 451, 999

Lenz P., Breger M., 2005, Comm. Asteroseis., 146, 5

Rodríguez E., Rolland A., López-González M. J., Costa V., 1998, A\&A, 338, 905 